\section{Ultrasound biomicroscopic findings of conventional and sutureless sclerotomy sites after 20-, 23-, and 25-G pars plana vitrectomy}

M Gutfleisch, M Dietzel, B Heimes, G Spital, D Pauleikhoff and A Lommatzsch
Department of Ophthalmology, St Franziskus Hospital, Muenster, Germany

Correspondence: $\mathrm{M}$ Gutfleisch, Department of Ophthalmology, St Franziskus Hospital, Hohenzollernring 74, Muenster, NRW 48145, Germany

Tel: 0049251933 080; Fax: 00492519330819 , E-mail: matthiasgutfleisch@ hotmail.com

Received: 20 October 2008 Accepted in revised form: 5 November 2009 Published online: 11 December 2009

\begin{abstract}
Purpose We examined sclerotomies that were performed using 20-G systems and non-stitch 23-G systems, one-step (23/1) and two-step procedures (23/2), as well as one-step 25-G systems for pars plana vitrectomy (PPV) with ultrasound biomicroscopy (UBM) to assess subconjunctival bleb formation, vitreous incarceration, and wound closure.

Methods This study is a prospective, nonrandomized, monocentre study comprising 40 patients; we examined sclerotomies using UBM after 20-G, 23/1-G, 23/2-G, and 25-G PPV in 10 patients each on days 1 and 30 postoperatively.

Results Subconjunctival bleb formation was not detected in any of the eyes on the first day after surgery in the $20-\mathrm{G}$ and $23 / 2-\mathrm{G}$ groups and was observed in $5(50 \%, 25 \mathrm{G})$ and 2 eyes (20\%, 23/1 G). By day 30 postoperatively, all blebs had spontaneously resolved. The sclerotomy tunnel could be detected by UBM at day 1 postoperatively in $10(100 \%, 20 \mathrm{G}), 9$ $(90 \%, 25 \mathrm{G}), 7(70 \%, 23 / 1 \mathrm{G})$, and 7 eyes $(70 \%$, $23 / 2 \mathrm{G})$. Thirty days postoperatively, it was present in $10(100 \%, 20 \mathrm{G}), 1(10 \%, 25 \mathrm{G}), 3$ $(30 \%, 23 / 1 \mathrm{G})$, and $1(10 \%, 23 / 2 \mathrm{G})$ eye. Vitreous incarceration occurred in $2(20 \%, 20 \mathrm{G}), 7$ (70\%, $25 \mathrm{G}), 4(40 \%, 23 / 1 \mathrm{G})$, and $2(20 \%, 23 / 2 \mathrm{G})$ eyes. Conclusions Anatomical closure of sclerotomies without subconjunctival bleb formation as seen on UBM was achieved at day 1 after 23/2-G and 20-G PPV and after 30 days postoperatively in all other non-stitch PPV techniques. Vitreous incarceration is
\end{abstract}

common after 25-G PPV. Non-stitch sclerotomies can frequently no longer be detected by UBM 30 days postoperatively. Eye (2010) 24, 1268-1272; doi:10.1038/eye.2009.291; published online 11 December 2009

Keywords: ophthalmological surgical procedures; vitrectomy; ultrasonic imaging; sclera

Introduction

Although conventional 20-G pars plana vitrectomy (PPV) has been the mainstay of vitreoretinal surgery in recent decades, it has been shown to have some disadvantages, such as prolonged postoperative discomfort or conjunctival scarring. Transconjunctival sutureless vitrectomy (TSV) has overcome many of the problems of 20-G PPV and is currently available in 25-G and 23-G systems. In general, one- and two-step techniques are used to insert the microcannula into the sclera. The 25-G PPV technique as introduced by Fujii et al. ${ }^{1}$ is performed in a one-step technique, 23-G PPV is also available as a one-step system (23/1 G). In this case, the microcannula is inserted into the sclera with a sharp trocar in one step. The original technique using a 23-G system as proposed by Eckhardt was established as a 23-G two-step (23/2-G) PPV system using a pressure plate to displace and stabilize the conjunctiva, creating a $30-45^{\circ}$ bevelled sclerotomy with a stiletto and then inserting a blunt trocar with a 
microcannula. ${ }^{2}$ Straight, oblique-parallel, and obliqueperpendicular incision techniques used to construct the scleral tunnel have also been described recently.

Ultrasound biomicroscopy (UBM) has been used to examine the architecture of sclerotomies in vivo after 25-G $\mathrm{G}^{3-6}$ and 20-G PPV. ${ }^{6-11}$ In addition to the typical intra-operative disadvantages such as difficulties faced while inserting the trocars, slipping out of cannulas, or flexible instruments, these studies have shown that wound closure from sutureless sclerotomies might be incomplete, subconjunctival blebs can form, and vitreous incarceration can develop. Furthermore, an increased risk of endophthalmitis after 25-G PPV has recently been reported compared with the conventional 20-G PPV. ${ }^{12-14}$ This might be due to inadequate wound closure that facilitates the influx of pathogens. In addition, vitreous becoming incarcerated in sclerotomy could be a risk factor for developing retinal detachment.

Recent studies have suggested that 25-G non-stitch PPV sclerotomies might be as effective, while reducing the typical problems associated with conventional PPV. In this prospective study, using UBM, we investigated whether 20-, 23/1-, 23/2-, or 25-G PPV techniques are of benefit with regard to conjunctival bleb formation, vitreous incarceration, and wound healing.

\section{Materials and methods}

A total of 40 consecutive patients who underwent PPV at the ophthalmology department at St Franziskus Hospital, Muenster, in 2007 were included in this study. This study followed the tenets of the Declaration of Helsinki. Approval from the appropriate ethics committee was obtained, and informed consent was acquired from all patients before surgery. Patients with different pathologies requiring PPV for treatment were included in this prospective study. Patients were excluded if they had undergone previous surgery or if a tamponade was required. Table 1 summarizes the indication for PPV.

All surgical procedures were performed by one of the authors (AL). Among the 40 patients, 25-G, 23/1-G, 23/2$G$, and 20-G three-port PPV systems were used for the procedure in 10 patients each.

All patients underwent surgery under general anaesthesia. The 20-G PPV was performed as a standard three-port PPV through a pars plana approach $3.5 \mathrm{~mm}$ from the limbus. The conjunctiva was opened $1 \mathrm{~mm}$ from the limbus, followed by scleral incisions $3.5-4 \mathrm{~mm}$ posterior to the limbus. We used a 1.4-mm sclerotomy blade (Bausch and Lomb, Rochester, NY, USA) penetrating the sclera straight and perpendicular to the scleral surface. In all cases, the vitreous within sclerotomies was removed using the vitrectomy cutter. Sclerotomies were closed with Vicryl 8.0 suture.
Table 1 Indications for surgery within the subgroups

\begin{tabular}{lcccc}
\hline & $20 G$ & $25 G$ & $23 / 1 G$ & $23 / 2 G$ \\
\hline Macular pucker & 3 & 9 & 6 & 7 \\
DRP & 5 & & 2 & \\
Vitreous haemorrhage due to & & & & \\
$\quad$ DRP & 1 & & 2 & 2 \\
BRVO & 1 & & & \\
$\quad$ Exudative AMD & & 1 & & 1 \\
$\quad$ Terson's syndrome & & & & 1 \\
\hline
\end{tabular}

Abbreviations: AMD, age-related macular degeneration; BRVA, branch retinal vein occlusion; DRP, diabetic retinopathy.

A 23-G three-port PPV setup was used for all 23-G cases. We used the Alcon 23-G one-step vitrectomy system (Alcon Laboratories Inc, Fort Worth, TX, USA) and the D.O.R.C. 23-G two-step vitrectomy system (Dutch Ophthalmic, Zuidland, The Netherlands). For both 23-G TSV PPV techniques, we used obliqueperpendicular sclerotomies by penetrating the conjunctiva and sclera perpendicular to the limbus at an angle of $30-45^{\circ}$ to the sclera, $3.5-4 \mathrm{~mm}$ posterior to the limbus. In $23 / 2 \mathrm{G}$, the conjunctiva was displaced with a pressure plate and the stiletto blade was inserted through the central opening of the blade. The blunt trocar was then inserted into each sclerotomy. In $23 / 1 \mathrm{G}$, the conjunctiva was displaced with a cotton tip and the microcannula was inserted with the sharp-edged trocar. After vitrectomy, microcannulas were removed and sclerotomies were covered by conjunctiva.

For the 25-G technique, we performed a direct incision with the trocar penetration straight and perpendicular to the scleral surface. We used the Bausch und Lomb 25-G vitrectomy system (Bausch and Lomb); otherwise, the surgical procedure was performed as described for the 23/1-G system.

No intra-operative complications developed in any of the patients. A complete eye examination was conducted including best-corrected visual acuity, tonometry (Goldmann), slit-lamp examination, ophthalmoscopy, and ultrasound biomicroscopic examinations at days 1 and 30 postoperatively. One sclerotomy was chosen for the ultrasound biomicroscopic examination, the temporal superior sclerotomy for right eyes and the nasal superior sclerotomy for left eyes to reduce examination times, patient discomfort, and possible pressure on the globe. Furthermore, there may be more manipulation at the aforementioned sclerotomy sites than for other sclerotomies in most right-handed surgeons from changing instruments, as speculated by Lopez-Guajardo et $a l,{ }^{4}$ who found that most blebs appeared in superior sclerotomies. Sclerotomy sites were examined by orienting the probe perpendicular and parallel to the limbus to intersect the entire sclerotomy tract. 


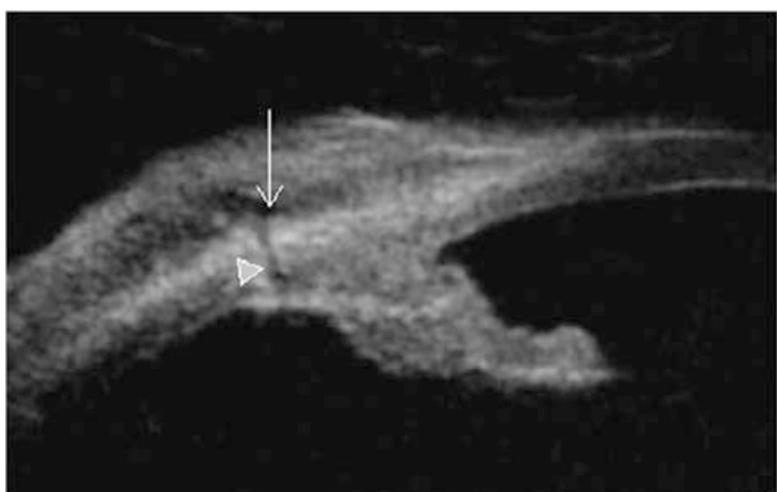

Figure 1 UBM of a sclerotomy (25G) with conjunctival bleb formation (arrow) and visible sclerotomy (arrowhead) at day 1 after surgery



Figure 2 UBM of a sclerotomy (25G) with resolved fluid (arrow) and anatomical closure as judged by UBM (arrowhead) day 30 postoperatively

Sclerotomies were examined using the VuMAX-II 35-50 Ultrasound Biomicroscopy System with a $50-\mathrm{Hz}$ transducer (Sonomed Inc, Lake Success, NY, USA). The UBM appearance on one image was evaluated independently by two investigators in a masked manner.

The main outcome measures were conjunctival bleb formation, echogenic appearance of the entire sclerotomy compared with the surrounding tissue, and vitreous incarceration. An echographically hyporeflective space between the conjunctiva and sclera was defined as conjunctival bleb formation (Figure 1). Healing was established when the echogenic appearance of the entire sclerotomy was similar to that of the surrounding tissue (Figure 2). Vitreous incarceration was diagnosed as echographically dense lines radiating from the interior part of the sclerotomy site into the vitreous cavity (Figure 3). Bleb formation was rated as present or not present, sclerotomies as healed or not healed, and vitreous incarceration as present or not present.

The statistical package SAS for Windows (Version 9.1, SAS, Institute Inc., Cary, NC, USA) was used for analysis. Descriptive comparisons were made using

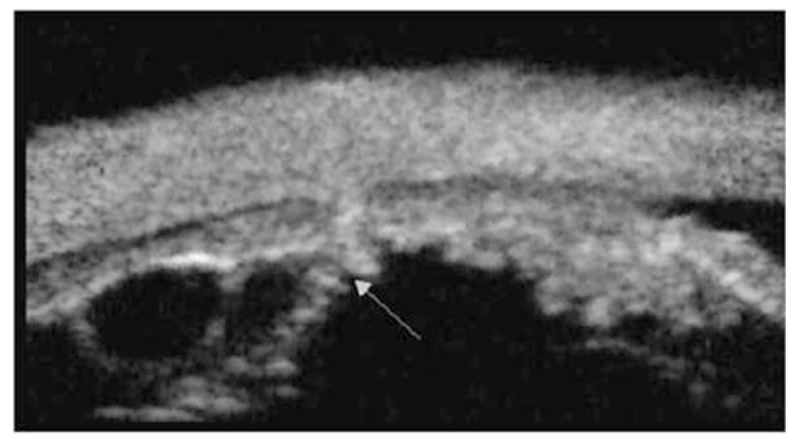

Figure 3 Vitreous incarceration (arrow) visible after 23/1G surgery at day 1 postoperatively, more posterior a vitreous base change

Fisher's exact test because of the small sample size. A $P$-value of $<0.05$ was considered statistically significant.

We certify that all applicable institutional and governmental regulations regarding the ethical use of human volunteers were followed during the research.

\section{Results}

Fisher' s exact test showed a significant difference between the vitrectomy systems with regard to subconjunctival bleb formation at day 1 postoperatively $(P<0.01)$. Subconjunctival bleb formation was not detected on the first day after surgery in any of the eyes after 20-G PPV. Bleb formation was detected in 5 out of 10 eyes (50\%) after 25-G PPV, in 2 out of 10 eyes (20\%) after 23/1-G PPV, and in none of the eyes after 23/2-G PPV (0\%, 23/2G). At the 30-day follow-up, all conjunctival blebs had spontaneously resolved.

One day after surgery, there was no significant difference between the vitrectomy systems regarding wound healing as judged by UBM $(P=0.26)$. The sclerotomy tunnel could be detected by UBM at day 1 postoperatively in all eyes $(100 \%, 20 \mathrm{G}), 9(90 \%, 25 \mathrm{G})$, $7(70 \%, 23 / 1 \mathrm{G})$, and 7 eyes $(70 \%, 23 / 2 \mathrm{G})$. Thirty days after surgery, the difference was significant between the vitrectomy systems $(P<0.01)$. The sclerotomy tunnel could still be identified in $10(100 \%)$ eyes after $20-\mathrm{G}$ PPV, in $1(10 \%)$ eye after 25-G PPV, in $3(30 \%)$ eyes after 23/1-G PPV, and in 1 (10\%) eye after 23/2-G PPV.

Vitreous incarceration approached statistically significant difference between the vitrectomy systems $(P=0.07)$ and was observed in $2(20 \%, 20 \mathrm{G}), 7(70 \%$, $25 \mathrm{G}), 4(40 \%, 23 / 1 \mathrm{G})$, and $2(20 \%, 23 / 2 \mathrm{G})$ eyes.

Postoperative ocular hypotension, defined as IOP $\leqslant 7 \mathrm{mmHg}$, was detected at day 1 in $0(0 \%, 20 \mathrm{G}), 2(20 \%$, $25 \mathrm{G}), 2$ eyes $(20 \%, 23 / 1 \mathrm{G})$, and in 1 eye $(10 \%, 23 / 2 \mathrm{G})$. Ocular hypotony was found more often in eyes with bleb formation $(3$ of $7 ; 43 \%)$ than in those without bleb formation $(2$ of $33 ; 6 \%)(P=0.03)$. Severe ocular 
hypotension, defined as IOP $\leqslant 5 \mathrm{mmHg}$ was detected at day 1 in 0 eyes $(0 \%, 20 \mathrm{G}, 23 / 2 \mathrm{G}), 1(10 \%, 25 \mathrm{G})$, and 2 eyes $(20 \%, 23 / 1 \mathrm{G})$. Postoperative ocular hypotony approached statistically significant difference between eyes with bleb formation $(2$ of $7 ; 29 \%)$ and those without bleb formation ( 1 of $33 ; 3 \%)(P=0.07)$.

In all, 2 of 5 eyes $(40 \%, 25 \mathrm{G})$ and 1 of 2 eyes (50\%, 23/1 G) with subconjunctival bleb formation had ocular hypotension 1 day postoperatively, whereas there was no bleb formation after 20- and 23/2-G PPV as mentioned before. We found no significant correlation between vitreous incarceration and bleb formation $(P=0.39)$.

No intra-operative complications were noted nor were additional procedures such as air filling or suture required in any of the patients. No other complications, such as retinal detachment or infectious endophthalmitis, were observed in any of the patients.

\section{Discussion}

Although conventional 20-G PPV may be of benefit, conjunctival scarring, inflammation, and discomfort are disadvantages of this technique. These typical features of conventional 20-G PPV can be avoided using TSV. However, disadvantages such as subconjunctival bleb formation are attributed to poor scleral closure. Moreover, vitreous incarceration in sclerotomies may increase complications, such as anterior retinal traction and subsequent retinal tears. In addition, both bleb formation and vitreous incarceration could result in a higher incidence of endophthalmitis after TSV.

In this study, 23-G non-stitch sclerotomies were performed in an oblique manner perpendicular to the limbus in all patients. This study suggests that sclerotomies performed in the 23/2-G PPV technique close better than do the other non-stitch techniques at day 1 after surgery according to the UBM appearance. Anatomical closure as judged by the UBM of sclerotomy sites is comparable with the results of conventional 20-G systems. After both techniques, subconjunctival bleb formation was not evident. However, after 1 month, there were no differences between sutureless sclerotomies regarding conjunctival bleb formation or wound healing according to UBM appearance, and sclerotomy sites could not be detected in most of the eyes.

No visible wound leakage with macroscopical accumulation of subconjunctival fluid was observed at the end of the operations. Therefore, the conjunctival elevation over the incision as seen in UBM might be attributed to different causes such as conjunctival manipulation during the operation or haemorrhage at the sclerotomy site. Mild wound leak could also manifest itself as conjunctival thickening over the sclerotomy site in the absence of an echo-free area under the conjunctiva.
Analysis of intraocular pressure 1 day postoperatively showed that eyes with conjunctival elevation had an ocular hypotony significantly more often than did those with normal or elevated IOD. These results suggest that conjunctival elevation and an echo-free area beneath the conjunctiva is a sign of intraocular leakage out of the eye.

Our observations show a significant difference between the vitrectomy systems with regard to subconjunctival bleb formation at the first day after surgery. After 20-G and 23/2-G PPV, no conjunctival bleb formation was noted at day 1 after surgery according to the UBM appearance in contrast to $23 / 1$ sclerotomies in which bleb formation was observed in $20 \%$ of the patients. It can be speculated that because of the angulated design of the separate stiletto as used in 23/2-G PPV, sclerotomies are more effectively constructed, even under difficult anatomical circumstances, such as deep-set eyes. After using 25-G PPV with direct incision techniques, incompletely closed sclerotomies were observed in 5 out of 10 patients as demonstrated by subconjunctival bleb formation in UBM. It has been previously shown that sclerotomies with angled incisions may seal quicker compared with direct incisions after 25-G PPV, because the sclera fibres are separated from each other rather than cut, resulting in better realignment after removing microcannulas. ${ }^{5,4}$ Anatomical and histopathological analysis in rabbits has shown that angled incisions were superior to straight incisions especially in $23-\mathrm{G}$ systems. ${ }^{15}$ At the end of the follow-up period, complete closure was achieved and no bleb formation was seen in UBM in all patients after TSV and conventional 20-G PPV.

In our study, sclerotomy could be detected on UBM on day 1 after surgery in most of the eyes and there was no significant difference between the incision techniques. One month after surgery, the sclerotomy site could not be detected by UBM in most eyes after non-stitch sclerotomy in contrast to 20-G sclerotomies. Several studies have shown that scleral wound healing may differ among sclerotomy techniques. Rizzo et $a l^{5}$ found that the oblique-parallel insertion achieved quicker and more complete sealing than did the obliqueperpendicular insertion in 25-G PPV. In another study, no differences were observed by UBM regarding the signs of healing after direct and oblique 25-G sclerotomies 15 days after surgery. ${ }^{4}$

In our study, vitreous incarceration was evident in $70 \%$ of the sclerotomy sites in UBM after 25-G PPV, in contrast to $20 \%$ after 23/2- and 20-G PPV and $40 \%$ after 23/1-G PPV. It can be speculated that vitreous entrapment and subconjunctival bleb formation facilitate intraocular infection. Several reports have raised the concern that the risk of endophthalmitis after 25-G PPV is higher than after conventional 20-G PPV. ${ }^{12-14}$ It can be 
speculated that the larger diameter of the $23-\mathrm{G}$ scleral tunnel as compared with the $25-\mathrm{G}$ direct incision increases bacterial influx, but the higher incidence of vitreous incarceration in 25-G PPV when performed with straight incision techniques could facilitate an intraocular infection. This study is limited by the small numbers of examined sclerotomies. Furthermore, we did not investigate oblique sclerotomies in 25-G PPV.

In conclusion, oblique-perpendicular sclerotomies in 23/2-G PPV may speed up wound closure with reduced leakage according to the UBM appearance of sclerotomies compared with oblique-perpendicular 23/1-G sclerotomies and 25-G straight sclerotomies. One month after surgery, no differences were found among non-stitch sclerotomy techniques regarding bleb formation and wound healing. Vitreous incarceration was observed more often after 25-G PPV with straight incisions. However, the safety of the different incision techniques regarding the incidence of serious complications such as endophthalmitis needs to be determined in future studies.

\section{Summary}

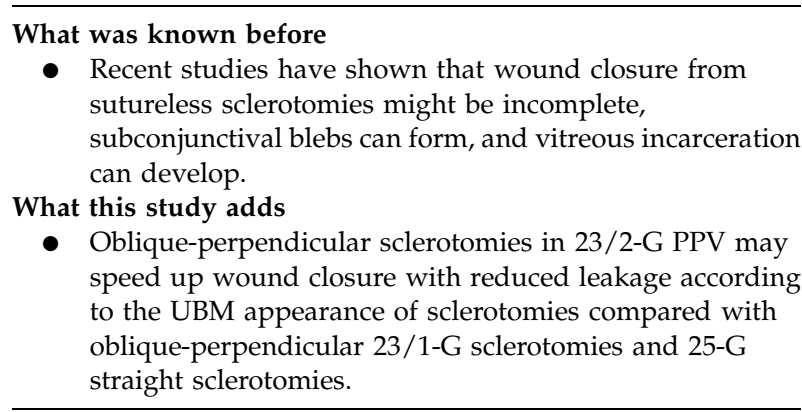

\section{Conflict of interest}

The authors declare no conflict of interest.

\section{References}

1 Fujii GY, De Juan Jr E, Humayun MS, Pieramici DJ, Chang TS, Awh C et al. A new 25-Gauge instrument system for transconjunctival sutureless vitrectomy surgery. Ophthalmology 2002; 109(10): 1807-1812; discussion 1813.

2 Eckardt C. Transconjunctival sutureless 23-Gauge vitrectomy. Retina 2005; 25(2): 208-211.
3 Kwok AK, Tham CC, Loo AV, Fan DS, Lam DS. Ultrasound biomicroscopy of conventional and sutureless pars plana sclerotomies: a comparative and longitudinal study. Am J Ophthalmol 2001; 132(2): 172-177.

4 Lopez-Guajardo L, Vleming-Pinilla E, Pareja-Esteban J, Teus-Guezala MA. Ultrasound biomicroscopy study of direct and oblique 25-Gauge vitrectomy sclerotomies. Am J Ophthalmol 2007; 143(5): 881-883.

5 Rizzo S, Genovesi-Ebert F, Vento A, Miniaci S, Cresti F, Palla M. Modified incision in 25-Gauge vitrectomy in the creation of a tunneled airtight sclerotomy: an ultrabiomicroscopic study. Graefes Arch Clin Exp Ophthalmol 2007; 245(9): 1281-1288.

6 Keshavamurthy R, Venkatesh P, Garg S. Ultrasound biomicroscopy findings of $25 \mathrm{G}$ transconjuctival sutureless (TSV) and conventional (20G) pars plana sclerotomy in the same patient. BMC Ophthalmol 2006; 6: 7.

7 Sabti K, Kapusta M, Mansour M, Overbury O, Chow D. Ultrasound biomicroscopy of sclerotomy sites: the effect of vitreous shaving around sclerotomy sites during pars plana vitrectomy. Retina 2001; 21(5): 464-468.

8 Theelen T, Verbeek AM, Tilanus MA, van den Biesen PR. A novel technique for self-sealing, wedge-shaped pars plana sclerotomies and its features in ultrasound biomicroscopy and clinical outcome. Am J Ophthalmol 2003; 136(6): 1085-1092.

9 Yeh PT, Yang CM, Yang CH, Huang JS. Cryotherapy of the anterior retina and sclerotomy sites in diabetic vitrectomy to prevent recurrent vitreous hemorrhage: an ultrasound biomicroscopy study. Ophthalmology 2005; 112(12): 2095-2102.

10 Hershberger VS, Augsburger JJ, Hutchins RK, Raymond LA, Krug S. Fibrovascular ingrowth at sclerotomy sites in vitrectomized diabetic eyes with recurrent vitreous hemorrhage: ultrasound biomicroscopy findings. Ophthalmology 2004; 111(6): 1215-1221.

11 Yang CM, Yeh PT, Yang CH. Intravitreal long-acting gas in the prevention of early postoperative vitreous hemorrhage in diabetic vitrectomy. Ophthalmology 2007; 114(4): 710-715.

12 Kwok AK, Tham CC, Lam DS, Li M, Chen JC. Modified sutureless sclerotomies in pars plana vitrectomy. $A m \mathrm{~J}$ Ophthalmol 1999; 127(6): 731-733.

13 Kunimoto DY, Kaiser RS. Incidence of endophthalmitis after 20- and 25-Gauge vitrectomy. Ophthalmology 2007; 114(12): 2133-2137.

14 Scott IU, Flynn Jr HW, Dev S, Shaikh S, Mittra RA, Arevalo JF et al. Endophthalmitis after 25-Gauge and 20-Gauge pars plana vitrectomy: incidence and outcomes. Retina 2008; 28(1): 138-142.

15 Singh RP, Bando H, Brasil OF, Williams DR, Kaiser PK. Evaluation of wound closure using different incision techniques with 23-Gauge and 25-Gauge microincision vitrectomy systems. Retina 2008; 28(2): 242-248. 